\title{
APLICAÇÃO DA PROGRAMAÇÃO LINEAR PARA MINIMIZAÇÃO DE PERDAS DE APARAS EM INDÚSTRIA METAL MECÂNICAA
}

\section{APPLICATION OF LINEAR PROGRAMMING TO MINIMIZE LOSSES OF CHIPS IN METALWORKING INDUSTRY}

\author{
Lucinéia Carla Loeblein ${ }^{1}$; Leoni Pentiado Godoy ${ }^{2}$; Loana Wollmann Taborda ${ }^{3}$; Diane \\ Cristina de Oliveira ${ }^{4}$ \\ ${ }^{1}$ Universidade Federal de Santa Maria - UFSM - Santa Maria - Brasil \\ lucineiacarla@yahoo.com.br \\ ${ }^{2}$ Universidade Federal de Santa Maria - UFSM - Santa Maria - Brasil \\ leoni_godoy@yahoo.com.br \\ ${ }^{3}$ Universidade Federal de Santa Maria - UFSM - Santa Maria - Brasil \\ loanataborda@yahoo.com.br \\ ${ }^{4}$ Sociedade Educacional Três de Maio - SETREM - Três de Maio - Brasil \\ diane_cta@yahoo.com.br
}

\begin{abstract}
Resumo
Os fornecedores de peças para montadoras de máquinas agrícolas têm uma enorme diversidade de itens, mas pequenos lotes devem ser fabricados com menor custo. Em um dos fornecedores do sul do Brasil, a cada semana há muitas ordens de fabricação de tubos com diferentes comprimentos a serem cortados. Portanto, este é um problema típico de otimização de corte de estoque, onde peças maiores precisam ser cortadas para a produção em tamanhos menores. Foi utilizada a programação linear para construir um modelo matemático apropriado para ser resolvido usando o aplicativo Solver do Excel® para controlar a produção, ou seja, os cortes de tubo. $O$ levantamento dos dados foi realizado por meio da análise dos relatórios do ERP - Enterprise Resource Planning da empresa. Realizou-se entrevista com colaboradores e também o levantamento ocorreu através dos roteiros de fabricação dos produtos. $O$ modelo foi aplicado a tubos de 6000 milímetros $(\mathrm{mm})$ de comprimento, 33,7 $\mathrm{mm}$ de diâmetro externo e espessura da parede do tubo de $3,35 \mathrm{~mm}$. Para demonstração neste artigo, utilizou-se a programação da produção de uma semana, na qual foram produzidos 28 componentes com diferentes comprimentos de tubo. Como resultados foram encontrados $1,21 \%$ de perda de material, em comparação com a percentagem de perda anterior que era entre 5 a 10\%, mostrando a importância da aplicação deste método no ganho de sustentabilidade da empresa.
\end{abstract}

Palavras-chave: Programação linear; sequenciamento de cortes; gestão de perdas. 


\section{Introdução}

Em virtude dos níveis de competitividade entre as empresas estarem cada vez mais acentuados, as indústrias tem a necessidade da busca constante pela melhoria dos processos com o objetivo de satisfazer os clientes e reduzir os custos de produção. A otimização dos recursos, no processo produtivo é estritamente necessário para a manutenção das indústrias no mercado. A Pesquisa Operacional, através da utilização de modelos matemáticos, pode contribuir nesse sentido, para encontrar soluções práticas na tomada de decisões no processo produtivo, a fim de minimizar custos, principalmente por meio da otimização de matéria-prima e mão de obra.

Os problemas de corte de estoque, que consiste no processo de corte de peças maiores para a produção de peças menores, ocorrem nas mais diversas situações, cada caso têm detalhes específicos que devem ser levados em consideração. Aproveitar a sobra de materiais é uma opção para inúmeras empresas, principalmente quando o custo da matéria-prima é significativo em relação ao valor final do produto. Além da questão econômica no reaproveitamento, ainda há a motivação pela sustentabilidade (CHERRI et al, 2011).

Várias pesquisas têm sido realizadas em relação ao problema de corte nas indústrias, com a utilização da Pesquisa Operacional para a solução dos problemas. Um destes trabalhos foi realizado por Cerqueira e Yanasse (2006), os quais apresentam alguns modelos de programação linear para a minimização de resíduos no processo de corte; Hoto et al (2003) apresentam o problema de corte de bobinas de aço; Beluzzo e Morabito (2005) apresentam um estudo de caso, em relação ao problema do corte de chapas de fibra de madeira reconstituída em uma indústria do setor. Além destes trabalhos, problemas de corte também podem ser encontrados em Bressan e Oliveira (2004), Campêlo et al (2009), Cherri et al (2011), Vianna e Nicola (2010), Franceschette (2009).

Nesse sentido, o presente estudo tem por objetivo a utilização da programação linear no sequenciamento do corte de tubos em uma indústria metal mecânica, a fim de minimizar as perdas na matéria-prima. A indústria caracteriza-se por ser uma pequena empresa do tipo Original Equipment Manufacturer - OEM, a qual destina seus produtos para outras empresas que montam as peças e vendem ao consumidor final. A exigência por parte dos clientes neste tipo de indústria é constante para a melhoria de processos e, consequentemente a redução dos custos. Neste sentido a programação linear pode trazer resultados positivos, com baixos investimentos.

O presente artigo está dividido em cinco seções, a primeira refere-se à introdução do estudo, a segunda seção apresenta o referencial teórico abordado no desenvolvimento do estudo, a terceira seção descreve a metodologia utilizada durante a pesquisa. A quarta seção descreve a análise dos resultados; e por fim, a quinta seção apresenta a conclusão do trabalho. 


\section{Referencial teórico}

\subsection{Pesquisa operacional}

De acordo com Andrade (2011), a Pesquisa Operacional iniciou durante a Segunda Guerra Mundial, por meio de equipes multidisciplinares que estudaram métodos para resolver problemas de operações militares. Pelo ótimo desempenho desta técnica, passou a ser utilizada por acadêmicos e empresários na resolução de problemas de administração. Pode-se afirmar que, não são necessários modelos matemáticos sotisficados, pois, quando os resultados mostram em valores a análise do processo produtivo, esses são mais eficientes que uma análise qualitativa.

Taha (2008) define a Pesquisa Operacional como uma ciência e também uma arte, é uma ciência, pois, utiliza técnicas matemáticas, e é uma arte porque a elaboração do modelo matemático depende da criatividade e da experiência dos profissionais. Portanto, sendo uma ciência aplicada, cujo objetivo pode-se dizer que é a melhoria da qualidade dos processos produtivos em organizações industriais, ou seja, em sistemas produtivos que se utilizam de qualquer tipo de recursos que fazem parte da produção.

Pesquisadores interdisciplinares utilizam técnicas e métodos científicos qualitativos para a tomada da decisão a fim de determinar a melhor utilização de recursos limitados e para a programação otimizada das operações de uma empresa (ANDRADE, 2011). Neste contexto, a interdisciplinaridade do campo de atuação da Pesquisa Operacional se estende da produção de matérias-primas e bens de consumo ao setor de serviços e às aplicações de interesse social como as relacionadas à saúde, à educação e à psico-sociologia, dessa forma procura abranger os diversos aspectos do mercado de trabalho.

A utilização de modelos matemáticos é uma das opções básicas para a tomada da decisão. A utilização dos mesmos atribui diversas vantagens em um processo de tomada de decisão (LACHTERMACHER, 2009). O modelo é uma representação aproximada da realidade através de equações matemáticas, a sua construção contribui para colocar as complexidades e possíveis incertezas de um problema em uma estrutura lógica, possibilitando uma análise mais ampla (WAGNER, 1986).

Problemas de tomada de decisão acontecem no dia-a-dia dentro das pequenas e grandes empresas. Mas, o difícil é os gestores decidirem qual a decisão a tomar, ou seja, o melhor caminho a seguir. Portanto, para o tomador de decisão é importante a adoção de métodos quantitativos, pois evita a utilização, somente da intuição e experiência, que poderá não ser a melhor decisão. As empresas que utilizam a Pesquisa Operacional e desenvolvem modelos de tomada de decisão estão na frente das concorrentes, o problema que ocorre em muitos casos, que nessas empresas as técnicas utilizadas são restritas, as quais não atendem a sua real necessidade (OLIVEIRA et al, 2010). 


\subsection{Programação linear}

A programação linear tem uma ampla aplicação e tornou-se uma das mais eficazes ferramentas para os estudos de gestão, como, organização de transportes, determinação de políticas de estoque, problemas de produção e misturas de componentes, entre outros casos. Entre os problemas a serem analisados, tem-se os de alocação dos recursos nas diversas atividades que devem ser realizadas. Na grande maioria dos casos estes recursos não são suficientes e existe a necessidade da melhor distribuição dos recursos disponíveis, de modo a atingir o melhor resultado para o objetivo proposto (ANDRADE, 2011).

Um problema de programação linear é um problema de programação matemática (LACHTERMACHER, 2009). E tem três componentes básicos: as variáveis de decisão, as quais se procura determinar; o objetivo, o qual é necessário otimizar e as restrições que a solução deve satisfazer (TAHA, 2008).

A programação linear é uma ferramenta essencial para ser usada na indústria, já existem diversos estudos que abordam a utilização destas em processos produtivos para evitar os desperdícios de matéria prima. É uma ferramenta que quando utilizada em termos financeiros, os resultados indicam redução nos custos do processo produtivo.

Diversas maneiras existem para encontrar a solução de um problema de programação linear, dentre elas estão a resolução gráfica, resolução analítica, além da utilização de softwares. Existem muitos softwares disponíveis que podem contribuir na resolução de problemas, dentre as ferramentas, as planilhas eletrônicas são as preferidas, pois além da facilidade de utilização, elas estão presentes em quase todas as empresas. As mais utilizadas são o Excel da Microsoft, o Lotus da Lotus/IBM e o Quattro-Pro da Corel, estas possuem as mesmas ferramentas, diferenciando apenas na forma do comando (LACHTERMACHER, 2009). Neste trabalho utilizou-se a planilha Excel da Microsoft, por meio da ferramenta Solver.

\subsection{Problema de corte de estoque}

De acordo com Poldi e Arenales (2004), padrão de corte é o modo como uma peça em estoque é cortada para a produção de peças menores. Bressan e Oliveira (2004) citam o exemplo do corte de barras, onde várias barras estão disponíveis para a produção de diversos itens, neste caso é necessário decidir quantas barras devem ser cortadas e como cortá-las.

E o problema de corte unidimensional engloba somente uma das dimensões importantes no processo de corte. Estes podem ocorrer no processo de bobinas de papel, tubos para produção de treliças, cortes de barras de aço com a mesma seção transversal (CHERRI, 2009).

Além do problema de corte unidimensional, também existem o problema de corte bidimensional e problema de corte tridimensional. No problema de corte bidimensional duas 
dimensões são relevantes (comprimento e largura) no processo de corte, estes problemas ocorrem principalmente em indústrias de placa de vidro, alumínio e madeira. E no problema de corte tridimensional três dimensões são relevantes no processo de corte (CHERRI, 2009). Neste estudo tem-se o problema de corte unidimensional.

\subsection{Programação da produção}

O principal desafio de um sistema de produção é o balanceamento entre a oferta e a procura. Tanto a demanda como a capacidade de produção são dimensões extremamente importantes, de forma que combiná-las é um desafio permanente da gerência de produção. Portanto, a programação linear é uma ferramenta que se pode utilizar para determinar a oferta e demanda dos produtos comercializados.

O processo de planejamento da produção exige um conjunto de decisões que devem ser tomadas para adaptar os recursos industriais da empresa de modo a satisfazer a demanda (SILVA FILHO, 2010). A programação linear pode auxiliar no planejamento da produção com a função de garantir que a produção ocorra eficazmente e produza produtos e serviços com qualidade e sem custos de desperdícios de material. Isto requer que os recursos produtivos estejam disponíveis: na quantidade adequada, no momento certo e no nível de qualidade desejado.

De acordo com Tubino (2009), a programação da produção é responsável por definir quando e quanto comprar, produzir ou montar de cada componente dos itens necessários para a composição dos produtos. Para isso são emitidas ordens de fabricação, ordens de compra, ordens de montagem. As atribuições da programação de produção podem ser divididas em três grupos: administração de estoques, sequenciamento e emissão e liberação de ordens.

No entanto, as empresas devem se preparar elaborando planos de longo prazo para dimensionamento de suas capacidades futuras, através de estudos de previsão de demanda. Além de que os seus objetivos devem ser formulados pelo planejamento estratégico, que serão definidos pela alta administração, com a finalidade de se fazer a previsão dos recursos. Estes devem ser distribuídos eficientemente para que não haja falhas no processo e desperdícios de matérias primas. Decisões devem ser tomadas pelos gestores da empresa e a programação linear é a ferramenta que encontra a maneira ideal ou mais eficiente de usar recursos limitados, principalmente na programação e controle da produção.

\subsection{Sequenciamento}

O sequenciamento da produção tem por objetivo gerar um programa de produção para os itens fabricados e montados que utilize de forma inteligente os recursos disponíveis, promovendo produtos com qualidade e preços baixos (TUBINO, 2009). Slack et al (2009), definem o 
sequenciamento como a ordem em que as tarefas devem ser executadas, para tanto existe um conjunto predefinido de regras que definem qual deve ser a ordem.

Uma destas regras é a restrição física dos materiais processados. Em alguns casos, as combinações de trabalho que chegam a uma operação podem determinar a prioridade dada aos trabalhos. Como exemplo, pode-se citar o corte de chapas de aço em indústrias metal-mecânicas, a chapa é cortada em determinado tamanho e formato, a chapa excedente será desperdiçada se não for utilizada para outro produto. Deste modo as operações que fisicamente se encaixam podem ser programadas para reduzir desperdícios (SLACK et al, 2009).

\subsection{Desperdícios}

No ambiente competitivo em que as empresas estão inseridas atualmente, estas devem produzir somente o necessário, na quantidade certa que o mercado precisa e na qualidade que os clientes exigem. As empresas que apresentam um alto índice de desperdício de uma forma ou outra contribuem para a redução da lucratividade das mesmas, assim, as atividades que não agregam valor ao produto devem ser reduzidas sistematicamente, não se admitindo mais qualquer forma de desperdícios. Deste modo, ressalta-se a importância da utilização da programação linear na mensuração dos desperdícios, desta forma é que os gestores conseguem tomar decisões coerentes usando dados e fatos e analisando valores correspondentes aos custos gerados pela má distribuição dos recursos limitados.

Conforme Shingo (1996), um dos princípios básicos do Sistema Toyota de Produção é a minimização dos custos. A origem dos lucros é vista da seguinte maneira: preço - custo = lucro, desta forma, a maneira de aumentar os lucros é por meio da redução dos custos, e um dos métodos para chegar a este resultado, é a redução total da perda. O objetivo dos fabricantes que desejam permanecer no mercado é a redução dos custos, para isso é necessário desenvolver a capacidade humana para realçar a criatividade e produtividade a fim de utilizar da melhor forma possível as máquinas e instalações para eliminar os desperdícios. Há a necessidade da busca constante pelo desperdício, pois o mesmo faz parte do processo produtivo, e não é facilmente notado (OHNO, 1997).

Assim é de fundamental importância que as empresas busquem sempre a eliminação do desperdício, entretanto, nem todos os gestores se utilizam da programação linear para socorrer sua empresa e eliminar os fatores geradores de custos. Normalmente, os gestores se utilizam de decisões qualitativas e deixam de lado, os modelos mais eficientes para distribuir o uso de recursos que são modelos matemáticos com a programação linear.

A melhoria nos processos pode ser realizada de duas maneiras, a primeira consiste em melhorar o produto e a segunda em melhorar os métodos de fabricação (SHINGO, 1996). Neste 
trabalho iremos trabalhar na melhoria dos métodos de fabricação com o objetivo da redução das perdas de matéria-prima.

Para que, as perdas possam ser identificadas, há necessidade de se conhecer de maneira detalhada todos os processos e operações que fazem parte do sistema produtivo. Segundo Bornia (1997) todo o trabalho realizado dentro de uma empresa pode ser classificado como trabalho que agrega valor ao produto e trabalho que não agrega valor ao produto. $O$ primeiro também denominado de trabalho efetivo refere-se a todas as atividades de transformação. O trabalho que não agrega valor, mas serve como suporte ao trabalho efetivo, é denominado de trabalho adicional. Entretanto, o trabalho que não agrega valor e, também, não é necessário ao trabalho efetivo, é denominado de desperdício. Dentro dessa categoria tem-se a produção de itens defeituosos, a movimentação desnecessária e a capacidade ociosa.

\section{Metodologia}

O estudo realizou-se em uma indústria fornecedora de peças para montadoras do ramo agrícola, seu principal foco é o desenvolvimento de componentes para colheitadeiras, plantadeiras e tratores, esta empresa está situada na região sul do Brasil, e sua especialidade são peças e partes em chapas e tubos de aço.

Esta pesquisa caracteriza-se por ser uma pesquisa aplicada, pois procura resolver o problema da grande quantidade de perdas de aparas no processo de corte de tubos na indústria pesquisada. Pois conforme Gil (2010), a pesquisa aplicada é aquela que busca resolver problemas, no ambiente em que os pesquisadores estão envolvidos.

Em relação às técnicas, as quais consistem nas atividades práticas realizadas durante a pesquisa (MARCONI e LAKATOS, 2011) foi utilizada a revisão bibliográfica do assunto a ser abordado, através de livros, revistas, internet, artigos; o levantamento dos dados foi realizado por meio de entrevistas não estruturadas com os colaboradores da empresa, análise dos relatórios do ERP - Enterprise Resource Planning da empresa e roteiros de fabricação dos produtos.

A produção nesta indústria caracteriza-se por possuir uma enorme diversidade de itens, os quais precisam ser produzidos em lotes mínimos ao menor custo. Cada semana há várias ordens de produção de tubos com tamanhos de cortes diferentes. O padrão de comprimento dos tubos de aço no mercado é $6000 \mathrm{~mm}$, para o processo produtivo tem-se a necessidade de cortar estes tubos em peças menores.

Atualmente a programação do corte de tubos é realizada de acordo com a data de entrega dos pedidos, os primeiros pedidos a serem entregues serão os primeiros a serem produzidos. Este método garante para a empresa, uma confiabilidade na data da entrega dos produtos, mas não permite trabalhar com a questão de otimização de recursos e de tempo. Portanto, este trabalho tem 
por objetivo, implantar uma programação de corte de tubos baseada na minimização de perdas de aparas e que atenda a data de entrega dos pedidos.

A programação do corte de tubos será realizada, por meio da utilização da programação linear, com o suporte do Microsoft Excel, através da ferramenta Solver.

\section{Análise e discussão dos resultados}

O planejamento da produção inicia a partir de um pedido do cliente através de uma ordem de compra. O cliente envia a ordem de compra, por meio do EDI - Intercâmbio Eletrônico de Dados, após sua importação é realizado a programação da produção, emitidas às ordens de produção de acordo com a data de entrega dos pedidos e encaminhadas para o processo produtivo.

O comprimento padrão de tubos de aço no mercado é de $6000 \mathrm{~mm}$. A indústria estudada tem a necessidade de cortar os tubos em diferentes tamanhos, para a fabricação de diversos itens de produção. Sem uma programação nos cortes, há grande perda de matéria-prima, as sobras dos tubos são encaminhadas para o estoque para uma futura reutilização ou então vendidas como sucata, a um preço muito inferior ao que foi pago. O problema desta pesquisa consiste em programar de forma mais eficaz o sequenciamento do corte de tubos para que haja menor perda de matéria-prima.

A indústria utiliza diversos diâmetros de tubo. A aplicação do modelo matemático iniciouse, nos componentes que usam como matéria-prima, o tubo 33,7 $\mathrm{mm}$ de diâmetro externo e 3,35 $\mathrm{mm}$ de espessura da parede do tubo, pois este é o tubo com maior consumo na indústria. O levantamento apontou a utilização deste tubo em 42 itens, com diferentes tamanhos de corte, ou seja, 42 comprimentos de cortes diferentes.

Para demonstração da resolução do problema neste trabalho, utilizou-se a programação da produção de corte de uma semana, considerada no trabalho como semana 01 . Na qual, tem se a produção de 28 itens diferentes com o tubo de 33,7 mm x 3,35 mm. Primeiramente analisaram-se como, os comprimentos dos tubos podem ser combinados para que haja menor perda de material. A Figura 1 apresenta uma aplicação da programação linear para atender a programação da semana 01. 
Figura 1 - Modelo de aplicação

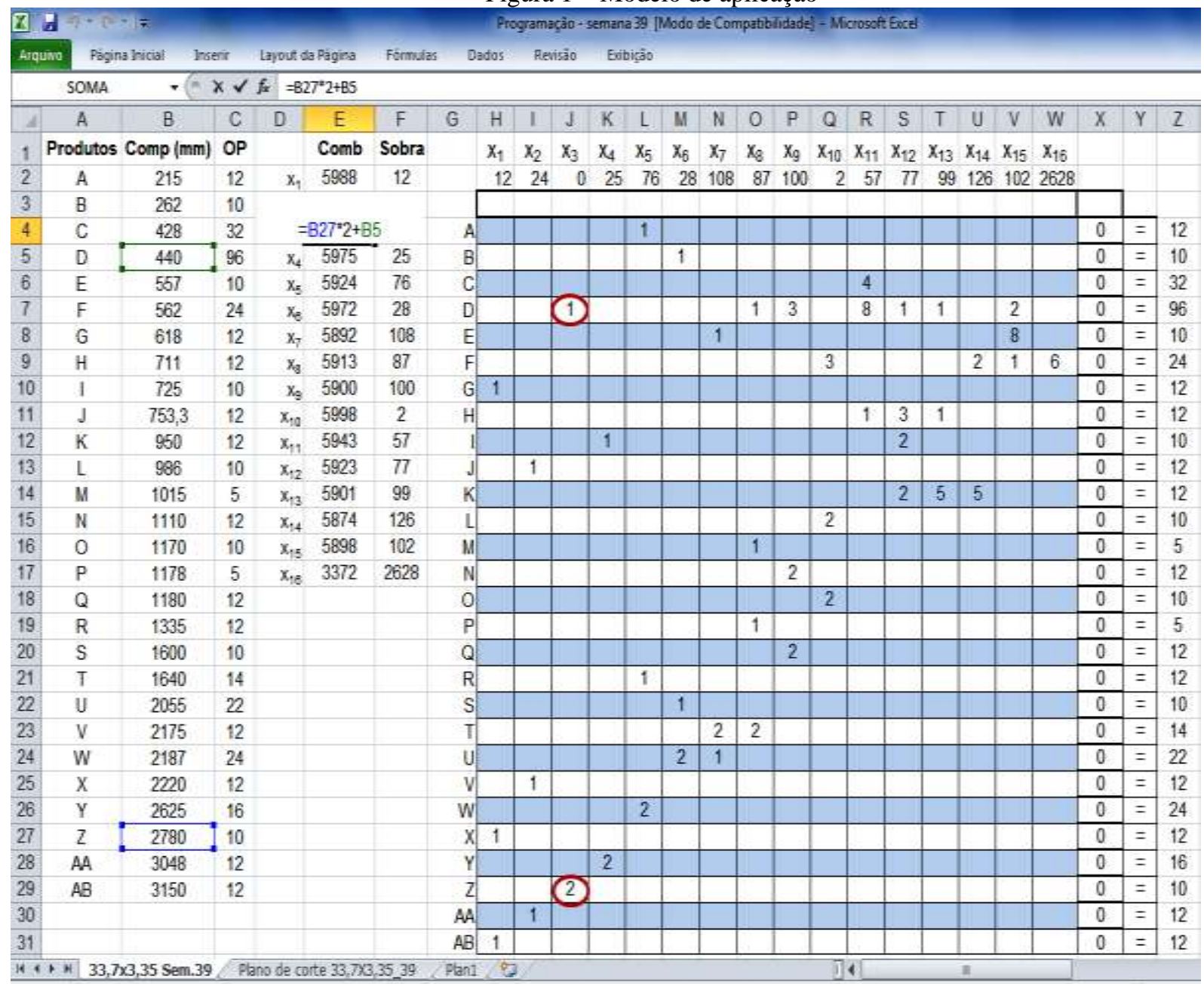

Fonte: Autoria própria (2012)

Os componentes a serem produzidos na semana 01 foram nomeados de $\mathrm{A}$ até $\mathrm{AB}$, conforme coluna A, da Figura 1. Os comprimentos em milímetros de cada componente estão demonstrados na coluna B e a quantidade em unidades de cada peça a ser produzida está demonstrada na coluna C.

A coluna E da Figura 1 apresenta as possíveis combinações para a otimização de matériaprima. O tamanho do tubo limita-se a $6000 \mathrm{~mm}$, como exemplo a combinação $\mathrm{x}_{3}$, é a soma do tubo $\mathrm{D}$ mais 2 vezes o tubo $\mathrm{Z}(440 \mathrm{~mm}+2 \times 2780 \mathrm{~mm}=6000 \mathrm{~mm})$, isso significa que cortando um tubo de $6000 \mathrm{~mm}$ nestas dimensões, atende-se a necessidade de 1 peça do item D e 2 peças do item Z, utilizou-se os $6000 \mathrm{~mm}$ do tubo, portanto não havendo sobra. A Figura 2 representa a combinação $\mathrm{x}_{1}$, na qual se tem os itens $\mathrm{G}$ com $618 \mathrm{~mm}$, X com $2220 \mathrm{~mm}$ e AB com $3150 \mathrm{~mm}$, e uma sobra de $12 \mathrm{~mm}$. 
Figura 2 - Demonstrativo de corte

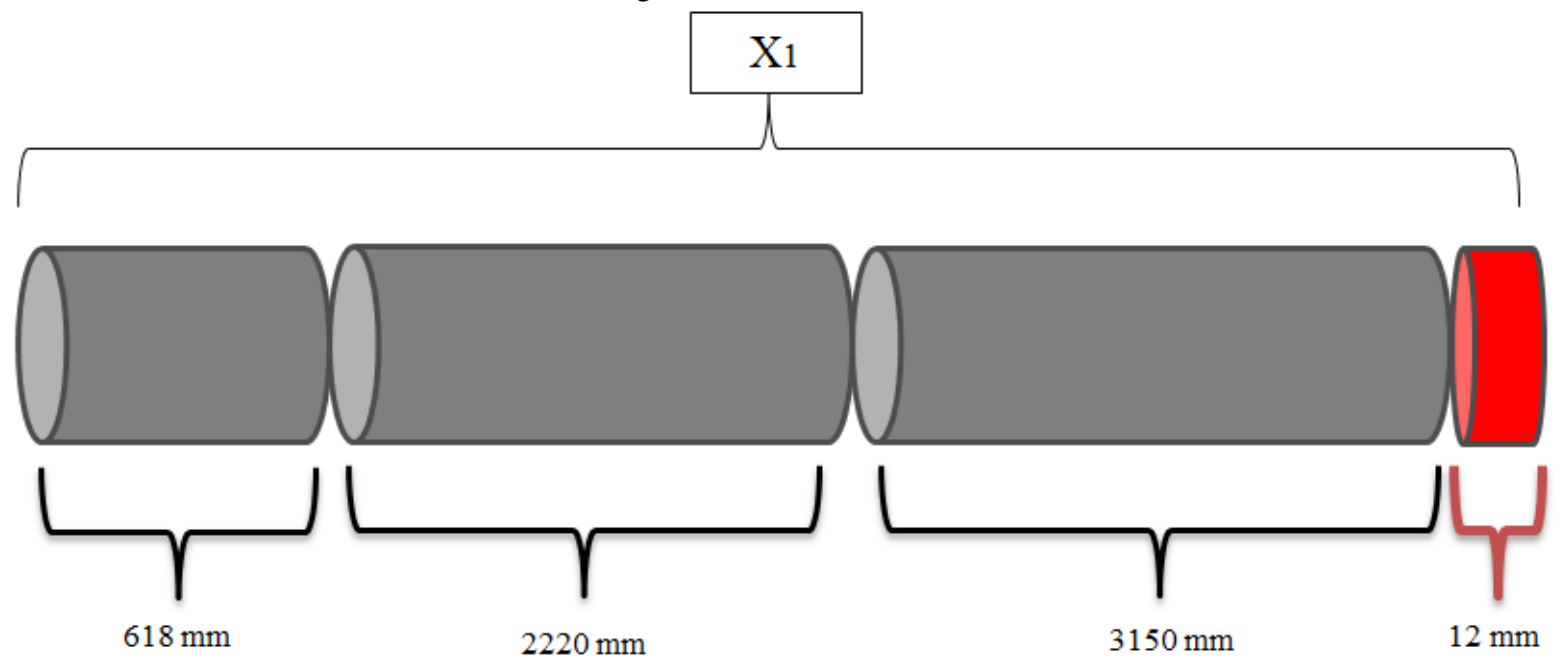

Fonte: Autoria própria (2012)

Na coluna F da Figura 1 tem-se a quantidade de tubo em milímetros que irá sobrar em cada combinação, e é esta sobra que se pretende minimizar. Portanto, a função objetivo é a minimização das perdas de aparas dos tubos nas possíveis combinações. Neste caso, encontrou-se 16 possíveis combinações.

Desse modo a função objetivo para a minimização de perdas de aparas é: $12 \mathrm{x}_{1}+24 \mathrm{x}_{2}+0 \mathrm{x}_{3}$ $+25 x_{4}+76 x_{5}+28 x_{6}+108 x_{7}+87 x_{8}+100 x_{9}+2 x_{10}+57 x_{11}+77 x_{12}+99 x_{13}+126 x_{14}+102 x_{15}+$ $2628 x_{16}$.

$\mathrm{E}$ as restrições:

$$
\begin{array}{ll}
\mathrm{x}_{5}=12 & 2 \mathrm{x}_{10}=10 \\
\mathrm{x}_{6}=10 & \mathrm{x}_{8}=5 \\
4 \mathrm{x}_{11}=32 & 2 \mathrm{x}_{9}=12 \\
\mathrm{x}_{3}+\mathrm{x}_{8}+3 \mathrm{x}_{9}+8 \mathrm{x}_{11}+\mathrm{x}_{12}+\mathrm{x}_{13}+2 \mathrm{x}_{15}=96 & \mathrm{x}_{5}=12 \\
\mathrm{x}_{7}+8 \mathrm{x}_{15}=10 & \mathrm{x}_{6}=10 \\
3 \mathrm{x}_{10}+2 \mathrm{x}_{13}+\mathrm{x}_{15}+6 \mathrm{x}_{16}=24 & 2 \mathrm{x}_{7}+2 \mathrm{x}_{8}=14 \\
\mathrm{x}_{1}=12 & 2 \mathrm{x}_{6}+\mathrm{x}_{7}=22 \\
\mathrm{x}_{11}+3 \mathrm{x}_{12}+\mathrm{x}_{13}=12 & \mathrm{x}_{2}=12 \\
\mathrm{x}_{4}+2 \mathrm{x}_{12}=10 & 2 \mathrm{x}_{5}=24 \\
\mathrm{x}_{2}=12 & \mathrm{x}_{1}=12 \\
2 \mathrm{x}_{2}+5 \mathrm{x}_{13}+5 \mathrm{x}_{14}=12 & 2 \mathrm{x}_{4}=16 \\
2 \mathrm{x}_{10}=10 & 2 \mathrm{x}_{3}=10 \\
\mathrm{x}_{8}=5 & \mathrm{x}_{2}=12 \\
2 \mathrm{x}_{9}=12 & \mathrm{x}_{1}=12
\end{array}
$$

Para a aplicação do Solver, as informações foram estruturadas na planilha nas células $\mathrm{H}$ até Z, conforme Figura 1. As variáveis de decisão encontram-se nas células H3:W3, são os valores que 
se busca encontrar para definir o valor ótimo da minimização das aparas, ou seja, quanto de cada combinação deve ser produzida para que se tenha uma menor perda de aparas. A função objetivo, que a solução busca otimizar nas células $\mathrm{H} 2$ :W2; as células H3 até X31 apresentam os coeficientes das restrições, as quais a solução deve atender. Após a estruturação dos dados aplicou-se os parâmetros do Solver.

Com a aplicação do Solver chegou-se a uma solução, na qual todas as restrições foram atendidas, esta solução se encontra nas colunas H3:W3 da Figura 3.

Figura 3 - Resultado da aplicação do Solver

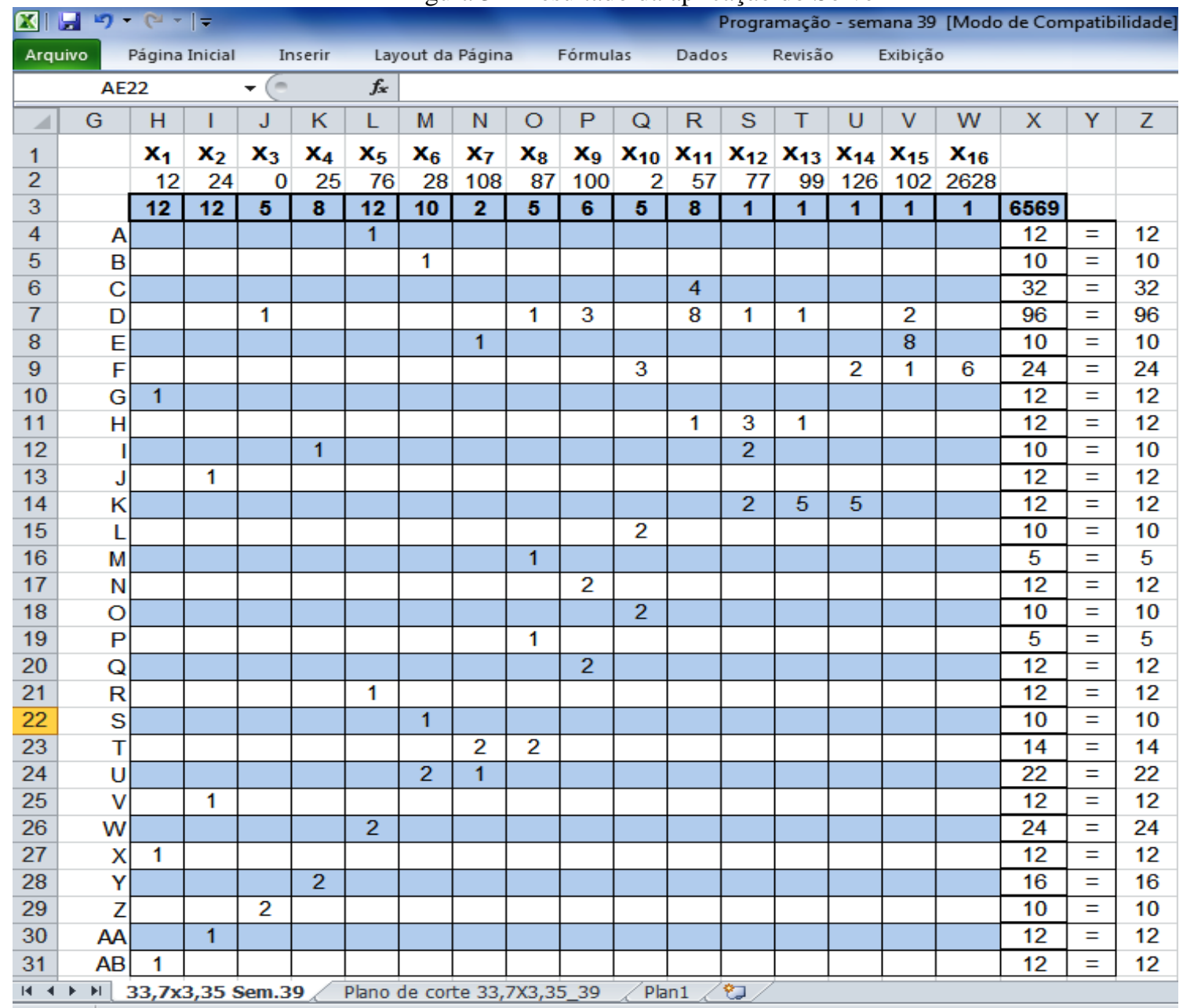

Fonte: Autoria própria (2012)

De acordo com a solução encontrada pelo Solver, para a semana 01, deve se cortar 12 tubos com a combinação $\mathrm{x}_{1}, 12$ tubos na combinação $\mathrm{x}_{2}, 5$ tubos na combinação $\mathrm{x}_{3} \mathrm{e}$ assim sucessivamente. Por meio de aplicação de fórmula, calculou-se uma perda de matéria-prima equivalente a $1,21 \%$, muito inferior a perda de $5 \%$ a $10 \%$ que ocorria antes da aplicação da programação linear.

Para os operadores da máquina de corte de tubos disponibilizou-se a programação de forma mais objetiva, conforme Figura 4, onde consta a quantidade a ser cortada de cada combinação e quanto sobra de cada tubo. 
Figura 4 - Plano de corte para produção

\begin{tabular}{|c|c|c|c|c|c|c|c|c|c|c|c|c|c|c|}
\hline & $0: 33,7$ & 3,35 & & & Data: & $21 / 0$ & 2011 & & Sema & a 01 & & & & \\
\hline & $\begin{array}{c}\text { Cód. da } \\
\text { peça }\end{array}$ & Comp & $(\mathrm{mm})$ & & $\begin{array}{c}\text { Cód. da } \\
\text { peça }\end{array}$ & Com & $(\mathrm{mm})$ & & $\begin{array}{c}\text { Cód. da } \\
\text { peça }\end{array}$ & Com & $(\mathrm{mm})$ & & $\begin{array}{c}\text { Cód. da } \\
\text { peça }\end{array}$ & Comp (mm) \\
\hline & $\mathrm{A}$ & & 5 & & $\mathrm{H}$ & & & & $\mathrm{O}$ & & & & $\mathrm{V}$ & 2175 \\
\hline & $\mathrm{B}$ & & 2 & & I & & & & $\mathrm{P}$ & & & & $\mathrm{W}$ & 2187 \\
\hline & $\mathrm{C}$ & & 8 & & $\mathrm{~J}$ & & & & $\mathrm{Q}$ & & & & $\mathrm{x}$ & 2220 \\
\hline & $\mathrm{D}$ & & 10 & & $\mathrm{~K}$ & & & & $\mathrm{R}$ & & & & $\mathrm{Y}$ & 2625 \\
\hline & $E$ & & 77 & & $\mathrm{~L}$ & & & & $\mathrm{~S}$ & & & & $Z$ & 2780 \\
\hline & $\mathrm{F}$ & & & & $\mathrm{M}$ & & & & $\mathrm{T}$ & & & & $\mathrm{AA}$ & 3048 \\
\hline & $\mathrm{G}$ & & 8 & & $\mathrm{~N}$ & & & & $\mathrm{U}$ & & & & $\mathrm{AB}$ & 3150 \\
\hline $\begin{array}{l}\text { Quant. } \\
\text { Barras }\end{array}$ & & & & & & Comp & mento & as bar & as (mm) & & & & & Sobra \\
\hline 12 & 3150 & 2220 & 618 & & & & & & & & & & & 12 \\
\hline 12 & 3048 & 2175 & 753,3 & & & & & & & & & & & 23,7 \\
\hline 5 & 2780 & 2780 & 440 & & & & & & & & & & & 0 \\
\hline 8 & 2625 & 2625 & 725 & & & & & & & & & & & 25 \\
\hline 12 & 2187 & 2187 & 1335 & 215 & & & & & & & & & & 76 \\
\hline 10 & 2055 & 2055 & 1600 & 262 & & & & & & & & & & 28 \\
\hline 2 & 2055 & 1640 & 1640 & 557 & & & & & & & & & & 108 \\
\hline 5 & 1640 & 1640 & 1178 & 1015 & 440 & & & & & & & & & 87 \\
\hline 6 & 1110 & 1110 & 1180 & 1180 & 440 & 440 & 440 & & & & & & & 100 \\
\hline 5 & 1170 & 1170 & 986 & 986 & 562 & 562 & 562 & & & & & & & 2 \\
\hline 1 & 950 & 950 & 725 & 725 & 711 & 711 & 711 & 440 & & & & & & 77 \\
\hline 1 & 950 & 950 & 950 & 950 & 950 & 711 & 440 & & & & & & & 99 \\
\hline 1 & 950 & 950 & 950 & 950 & 950 & 562 & 562 & & & & & & & 126 \\
\hline 8 & 440 & 440 & 440 & 440 & 440 & 440 & 440 & 440 & 428 & 428 & 428 & 428 & 711 & 57 \\
\hline 1 & 557 & 557 & 557 & 557 & 557 & 557 & 557 & 557 & 440 & 440 & 562 & & & 102 \\
\hline 6 & 562 & USAF & RETA & HOS & & & & & & & & & & \\
\hline
\end{tabular}

Fonte: Autoria própria (2012)

Acompanhou-se a implantação do sequenciamento de corte na produção na semana 01, para verificação da correta execução das atividades e orientação aos colaboradores. Ao mesmo tempo em que se conferiu o comprimento dos retalhos dos tubos. Verificou-se, uma determinada resistência por parte dos operadores na execução das atividades conforme a Figura 4, mas com a explicação e o resultado após a conclusão da operação, mostraram-se motivados com este novo modelo de sequenciamento de corte de tubos.

\section{Conclusão}

O trabalho apresenta a viabilidade da aplicação da Pesquisa Operacional - Programação Linear em pequenas empresas, pode-se dizer que havendo pessoas capacitadas para a aplicação e acompanhamento a programação linear é uma ferramenta eficiente para a melhoria de processos e redução de custos, sem a necessidade de grandes investimentos financeiros. A utilização do MS Excel, é uma prática comum em indústrias, e a aplicação da ferramenta Solver pode contribuir para a manutenção das indústrias em um mercado tão competitivo, onde a melhoria de processos é uma busca constante. 
O objetivo do trabalho foi a implantação do sequenciamento do corte de tubos a fim de reduzir as perdas na matéria-prima, por meio da programação linear. Este objetivo foi atingido ao se chegar a uma combinação de corte de tubos possível, em que todas as restrições foram atendidas e a sua efetiva implantação no processo produtivo.

Além de possibilitar o melhor aproveitamento da matéria-prima e evitar que essa matériaprima de boa qualidade se torne sucata, irá diminuir o estoque de retalhos de tubos e consequentemente a redução dos custos de produção e, aumento nos lucros. Para Ragsdale (2009) no ambiente comercial e competitivo de hoje, é cada vez mais importante garantir que os recursos limitados de uma empresa sejam usados da maneira mais eficiente possível. $\mathrm{O}$ autor mostra ainda que, isso envolve determinar como alocar os recursos de maneira a maximizar os lucros ou minimizar custos. Por isso, pode-se dizer que a programação linear é uma ferramenta de otimização que se bem aplicada é eficaz e eficiente na distribuição de recursos na empresa.

Referente ao cunho acadêmico o estudo foi de grande valia, pois resolveu um problema de corte de tubos na empresa e houve a implantação desta metodologia, o que proporcionou ao especialista melhor oportunidade de aprendizado com a aplicação prática da pesquisa operacional no meio industrial.

Ficou evidenciado neste artigo que o tema estudado é importante e que demanda esforços no sentido de melhorar a qualidade do produto final e reduzir perdas e custos de produção. Como sugestão de futuros trabalhos sugere-se a verificação do tempo de setup de máquina com o sequenciamento do corte de tubos, além da aplicação do sequenciamento de corte para os demais diâmetros de tubos.

\begin{abstract}
Suppliers of parts for farm machinery manufacturers have a huge variety of items and small batches should be manufactured with lower cost. In one of these suppliers in south of Brazil, every week there are many orders of manufacturing of tubes with different lengths to be cut. So this is a typical optimization problem, cutting stock, where larger pieces need to be cut for the production of smaller parts. Linear programming was used to construct a mathematical model solved by using suitable for the application Solver Excel ${ }^{\circledR}$ to control the production, or, the sections of pipe. Data collection was conducted through analysis of reports from the Enterprise Resource Planning (ERP) of company, interviews with employees and through the scripts of their manufacturing. The model was applied to tubes $6000 \mathrm{~mm}$ length, 33,7 $\mathrm{mm}$ in outside diameter and of $3.35 \mathrm{~mm}$ wall thickness tube. To demonstrate this article, used to program the production of a week in which components 28 were produced with different lengths of pipe. The results were found 1,21\% loss of material, compared to the percentage that were previously used between $5-10 \%$, which shows the importance of this method in the gain of sustainability of the company.
\end{abstract}

Key-words: Linear programming; Sequencing of cuts; Management losses. 


\section{Referências}

ANDRADE, E. L. Introdução à Pesquisa Operacional: Métodos e Modelos para Análise de Decisões. 4 ed. Rio de Janeiro: LTC, 2011.

BELUZZO, L.; MORABITO, R. Otimização nos padrões de corte de chapas de fibra de madeira reconstituída: um estudo de caso. Revista Pesquisa Operacional, 25, p. 391-415, 2005.

BORNIA, A. C. Mensuração das perdas dos processos produtivos: uma abordagem metodológica de controle interno (Tese de doutorado em Engenharia de Produção). UFSC, 1995.

BRESSAN, G. M.; OLIVEIRA, A. R. L. Reordenamento eficiente das colunas básicas na programação de lotes e cortes. Revista Pesquisa Operacional, 24, p. 323-337, 2004.

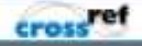

CÂMPELO, M.; CAMPOS, V.; CORRÊA, R. Um algoritmo de planos-de-corte para o número cromático fracionário de um grafo. Revista Pesquisa Operacional, 29, p. 179-193, 2009.

\section{cross'}

CERQUEIRA, G. R. L.; YANASSE, H. H. Linear Programming Models for the One-Dimensional Cutting Stock Problem. INPE ePrint, 1, 2006.

CHERRI, A. C.; ALEM, D. J. J.; SILVA, I. N. Inferência Fuzzi para o problema de corte de estoque com sobras aproveitáveis de material. Revista Pesquisa Operacional, 31, p. 173-194, 2011.

\section{cross ref}

CHERRI, A. C. Algumas extensões do problema de corte de estoque com sobras de material aproveitáveis. 2009. 115 f. Tese (Doutorado em Ciências - Ciências de Computação e Matemática Computacional) - Universidade de São Paulo, São Paulo, 2009.

FRANCESCHETTE, C. Uma abordagem heurística para o problema de corte guilhotinado bidimensional aplicado a uma situação de canibalização. In Simpósio de Engenharia de Produção, XVII, 2010, Bauru. Anais... Bauru, 2010.

GIL, A. C. Como elaborar projetos de pesquisa. 5 ed. São Paulo: Atlas, 2010.

HOTO, R.; MACULAN, N., MARQUES, F.; ARENALES, M. Um problema de corte com padrões compartimentados. Revista Pesquisa Operacional, 23, p 169-187, 2003.

\section{cross ${ }^{\text {ref }}$}

LACHTERmaCHer, G. Pesquisa Operacional na tomada de decisões. 4 ed. São Paulo: Pearson Prentice Hall, 2009.

MARCONI, M. A.; LAKATOS, E. M. Metodologia do trabalho científico. 7 ed. São Paulo: Atlas, 2011.

OHNO, T. O Sistema Toyota de Produção: além da produção em larga escala. Porto Alegre: Artes Médicas, 1997.

OLIVEIRA, F. F.; SILVEIRA, R. R. \& FERREIRA, R. J. P. Pesquisa Operacional como apoio na tomada de decisões em Gestão da Produção. XVII Simpep, 2010.

POLDI, K. C.; ARENALES, M. N. O problema de corte de estoque unidimensional multiperíodo. Revista Pesquisa Operacional. 30, p. 153-174, 2010.

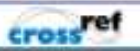

RAGSDAlE, C. T. Modelagem e Análise de Decisão. Ed. Revisada. São Paulo: Ed. Cengage Learning.2009.

SHINGO, S. O Sistema Toyota de Produção: do ponto de vista da Engenharia de Produção. 2 ed. Porto Alegre: Artes Médicas, 1996.

SILVA FILHO, O. S. Gerando planos de produção através de um problema linear quadrático gaussiano com restrições nas variáveis de decisão. Revista Pesquisa Operacional. 30, p. 99-124, 2010. 
SLACK, N.; CHAMBERS, S. \& JOHNSTON, R. Administração da produção. 3. ed. São Paulo: Editora Atlas S.A., 2009.

TAHA, H. Pesquisa Operacional: uma visão geral. 8. ed. São Paulo: Pearson Prentice Hall, 2008.

TUBINO, D. F. Planejamento e Controle da Produção: teoria e prática. 2. ed. São Paulo: Editora Atlas S. A., 2009.

VIANNA, A. C. G.; NICOLA, A. C. C. Estudo comparativo entre a abordagem em grafo e/ou e o algoritmo de Wang para o problema de corte bidimensional. In Simpósio de Engenharia de Produção, XVII, 2010, Bauru. Anais... Bauru, 2010.

WAGNER, H. M. Pesquisa operacional. 2. ed. Rio de Janeiro: Prentice Hall do Brasil, 1986.

\section{Dados dos autores:}

Nome completo: Lucinéia Carla Loeblein

Filiação institucional: Universidade Federal de Santa Maria (UFSM)

Departamento: Departamento de Engenharia de Produção e Sistemas (DEPS)

Função ou cargo ocupado: Mestranda - Bolsista Capes

Endereço completo para correspondência: Departamento de Engenharia de Produção e Sistemas, Centro de Tecnologia, UfSM, Campus Camobi, Bairro Camobi, Santa Maria, Rio Grande do Sul, Brasil, CEP 97105-900

Telefone para contato: (55) 3220 - 8442

e-mail: lucineiacarla@yahoo.com.br

\section{Nome completo: Leoni Pentiado Godoy}

Filiação institucional: Universidade Federal de Santa Maria (UFSM)

Departamento: Departamento de Engenharia de Produção e Sistemas (DEPS)

Função ou cargo ocupado: Professora do Programa de Pós-Graduação em Engenharia de Produção

Endereço completo para correspondência: Departamento de Engenharia de Produção e Sistemas, Centro de Tecnologia, UFSM, Campus Camobi, Bairro Camobi, Santa Maria, Rio Grande do Sul, Brasil, CEP 97105-900

Telefone para contato: (55) 3220 - 8442

e-mail: leoni_godoy@yahoo.com.br

\section{Nome completo: Loana Wollmann Taborda}

Filiação institucional: Universidade Federal de Santa Maria (UFSM)

Departamento: Departamento de Engenharia de Produção e Sistemas (DEPS)

Função ou cargo ocupado: Mestranda 
Endereço completo para correspondência: Departamento de Engenharia de Produção e Sistemas, Centro de Tecnologia, UFSM, Campus Camobi, Bairro Camobi, Santa Maria, Rio Grande do Sul, Brasil, CEP 97105-900

Telefone para contato: (55) $3220-8442$

e-mail: loanataborda@yahoo.com.br

Nome completo: Diane Cristina de Oliveira

Filiação institucional: Sociedade Educacional Três de Maio (SETREM)

Departamento: Departamento de Engenharia de Produção

Função ou cargo ocupado: Estudante

Endereço completo para correspondência: Avenida Santa Rosa, 2405, Três de Maio, Rio Grande do Sul, CEP 98910-000

Telefone para contato: (55) 3535 - 4600

e-mail:diane_cta@yahoo.com.br

Recebido em: 03/12/2012

Aceito em: 23/01/2013 